\title{
Nosocomial infections: A further assault on patients in a high-volume urban trauma centre in South Africa
}

\author{
A J Dell, BSc, MB ChB, MMed (Surg), PhD, FCS (SA); P H Navsaria, MB ChB, MMed (Surg), FCS (SA), FACS Trauma Surgery; \\ S Gray, BSc Hons, MB BCh; J C Kloppers, MB ChB, MMed (Surg), FCS (SA), MPhil (Surg), Cert Gastroenterology (SA) Surg \\ Trauma Centre, Department of Surgery, Groote Schuur Hospital and Faculty of Health Sciences, University of Cape Town, South Africa
}

Corresponding author: A J Dell (angelajdell@gmail.com)

\begin{abstract}
Background. Hospital-acquired infections (HAIs) are a major cause of morbidity and mortality. Surgical site infection (SSI) rates are reported to range from $2.5 \%$ to $41 \%$. HAI increases the risk of death by $2-11 \%$, and three-quarters of these deaths are directly attributable to SSIs. Objectives. To determine the incidence of HAI and to identify risk factors amenable to modification with a resultant reduction in infection rates.

Methods. An analysis of HAIs was performed between January and April 2018 in the trauma centre surgical wards at Groote Schuur Hospital, Cape Town, South Africa.

Results. There were 769 admissions during the study period. Twenty-two patients $(0.03 \%)$ developed an HAI. The majority were men, and the mean age was 32 years (range 18 - 57). The mean length of hospital stay (LoS) was 9 days, higher than the mean LoS for the hospital of 6 days. Fourteen patients underwent emergency surgery, 3 patients underwent abbreviated damage control surgery, and 9 patients were admitted to the critical care unit. Most patients with nosocomial sepsis were treated with appropriate culture-based antibiotics (82\%). Four patients were treated with amoxicillin/clavulanic acid presumptively prior to culture and sensitivity results, after which antibiotic therapy was tailored. All but 1 patient received antibiotics.

Conclusions. A combination of measures is required to prevent trauma-related infections. By determining the incidence of nosocomial infections in our trauma patients, uniform policies to reduce infection rates further could be determined. Our low incidence of infection may be explained by established preventive care bundles already in place.
\end{abstract}

S Afr Med J 2020;110(2):123-125. https://doi.org/10.7196/SAMJ.2020.v110i2.14243

Hospital-acquired infections (HAIs) affect more than two million patients annually in the USA. Similarly, surgical site infections (SSIs) are a major cause of morbidity and even mortality, particularly in developing countries. ${ }^{[1-3]}$ The World Health Organization (WHO) predicted that post-traumatic mortality is increasing and will be a major cause of death by $2020 .^{[4,5]}$ Globally, SSI rates have been reported to range from $2.5 \%$ to $41.0 \% .^{[1,2]}$ SSI is the third most common nosocomial infection and the most common HAI among surgical patients. ${ }^{[6]}$ Patients whose surgery was complicated by an SSI have a $2-11 \%$ increased risk of death, and three-quarters of these deaths are directly attributable to SSIs. ${ }^{[7]}$ Clinically, SSIs occur in the part of the body where surgery took place. SSI occurs within 30 days after surgery, and up to a year after the procedure in the case of an implant. ${ }^{[8,9]}$

Worldwide, trauma is the leading cause of death among adults between 25 and 44 years of age. ${ }^{[10]}$ Patients who survive the immediate injury and are admitted to the intensive care unit (ICU) are vulnerable to HAI, with pneumonia being the most common infection if an injury to the respiratory system or intubation has occurred. Both pro- and anti-inflammatory responses are involved in the post-traumatic pathological process, and they increase the risk of acute respiratory distress syndrome, sepsis and multiple organ failure. Early prevention of the development of sepsis following trauma can reduce both sepsis and multiple organ dysfunction syndrome, which can improve patient outcomes. ${ }^{[1]}$ Preventive efforts should therefore focus on both trauma-related infection/sepsis and subsequent organ dysfunction. Infection prevention strategies currently in place include surgical management and the administration of pharmaceuticals (prophylactic antibiotics, pre-emptive antibiotics, tetanus vaccination and immunomodulatory interventions). ${ }^{[1]}$ In addition, enhanced recovery after surgery (ERAS) and enhanced recovery after trauma surgery (ERATS) protocols have resulted in improved postoperative outcomes.

Identifying independent risk factors for nosocomial infection in trauma patients is a difficult task. However, multivariate analysis has shown that post-traumatic sepsis is associated with male gender, existing comorbidities, the presence of shock, type of trauma (blunt or penetrating), prolonged ventilation, wound classification, the use of prophylactic antibiotics, spinal cord injury, high Injury Severity Score, greater number of injuries, number of packed red blood cells transfused, number of operative procedures, and laparotomy. ${ }^{[12,13]}$ Patients with any of these risk factors or with features of sepsis should be actively investigated until the source is identified and managed and the patient improves clinically. ${ }^{[14]}$

\section{Objectives}

To determine the incidence of HAI and to identify risk factors amenable to modification with a resultant reduction in infection rates.

\section{Methods}

A prospective descriptive analysis of the incidence of HAI was performed over the 4-month period 1 January - 30 April 2018 in the trauma centre at Groote Schuur Hospital, Cape Town, South Africa (SA). All patients admitted to the trauma surgical wards who developed SSI post trauma as well as any nosocomial infections were 
included in the study. Their demographic data and risk factors for sepsis were analysed; these included past medical history, substance and medication use, and smoking history. Data were captured in a password-protected Excel spreadsheet, 2013 version (Microsoft, USA). Antibiotic administration and whether this adhered to unit policies was recorded. University of Cape Town Human Research Ethics Committee approval was obtained (ref. no. 856/2017).

\section{Results}

There were 769 admissions to the trauma wards during the 4-month study period. During this time, 22 patients $(0.03 \%)$ were identified with HAIs, of which 12 and 5 were superficial and deep SSIs, respectively, 2 were urinary tract infections (UTIs), 4 were lower respiratory tract infections (LRTIs) and 1 was post-traumatic meningitis. Three patients developed both an SSI and LRTI.

The mean age was 32 years (range 18 - 57), and of the patients who developed nosocomial infections, 19 were men and 3 were women, in keeping with the gender trend in trauma populations. There were no deaths associated with HAI.

Three hundred and ninety-four surgical procedures were performed during the 4-month study period. The mean length of stay (LoS) during the study period was 6 days, with long staying outliers affecting this number. Patients who developed HAI had a mean LoS of 9 days (range 2 - 35).

Two (9\%) of the 22 patients were known to be HIV-positive. One of these was on antiretroviral treatment (ART) with a lower than detectable viral load, and the other had defaulted from ART and did not have a recent $\mathrm{CD} 4+$ count or viral load. The rest of the patients denied being HIV-positive, and they were not specifically tested as a result of current counselling and practices in SA.

Five patients were classified as shocked on admission, and 4 of these received a massive blood transfusion. Fourteen patients underwent emergency surgery, 3 underwent abbreviated damage control surgery, and 9 were admitted to an ICU. Most patients admitted to substance use: 10 patients smoked, 4 used ethanol regularly, and 3 were using recreational drugs (heroin and methaqualone). Wounds were classified as follows: clean $(n=1)$, clean-contaminated $(n=9)$, contaminated $(n=3)$ and dirty $(n=9)$.

All but one of the patients were treated with antibiotics. In this case, an Acinetobacter culture-positive UTI was successfully managed with removal of the indwelling urinary catheter. The first-line therapy in 14 patients (67\%) was amoxicillin and clavulanic acid, and 4 patients were escalated to piperacillin/tazobactam and amikacin.

As part of the local antibiotic policy, 5 patients were treated de novo with second-line therapy (piperacillin/tazobactam). The patient with post-traumatic meningitis was treated with meropenem as dictated by protocol for meningitis related to penetrating injuries. Most patients with nosocomial sepsis $(82 \%)$ were treated with appropriate culture-based antibiotics. Four patients were treated with amoxicillin and clavulanic acid presumptively prior to culture and sensitivity results, after which antibiotic therapy was changed or stopped as appropriate. Table 1 and Fig. 1 demonstrate mean septic parameter values of the study population.

\section{Discussion}

HAIs are an important cause of morbidity and mortality, and increase the cost of healthcare. Trauma patients are susceptible to developing infections as a result of the systemic inflammatory response and multiorgan dysfunction that result from severe injury. ${ }^{[10]}$ Quantifying the incidence of HAI is a starting point in identifying and understanding factors that may predispose trauma patients to these infections. The incidence of $0.03 \%$ over 4 months in the present study seems very low compared with previously reported figures of between $2.5 \%$ and $41 \% .^{[1,2]}$ In patients who developed infections, the mean LoS increased marginally from 6 to 9 days. Increased LoS adds to the overall cost of patient care, which is further compounded by additional medication, including antibiotics, and tests carried out during treatment. Our trauma patients tend to be young, previously healthy males, which could have provided some protection against the development of HAI compared with older patients with multiple comorbidities.

The results of the present study show that our unit adheres to current antibiotic stewardship policies, with $82 \%$ of patients being treated with appropriate culture-based antibiotics. There were four instances where antibiotics were started empirically based on the clinical suspicion of infection, but with daily review of medication charts, antibiotic stewardship ward rounds and critical discussion surrounding rationalising antibiotic choices, treatment was adjusted as appropriate. With the development of pan-resistant strains of so-called superbugs, it is more important than ever to ensure that healthcare institutions take responsibility for their role in curbing this pandemic. A. baumanni and methicillin-resistant Staphylococcus aureus resistant to most antimicrobials have emerged, especially in ICUs. ${ }^{[15]}$ Early identification and treatment of infection with these organisms will help prevent their spread in the hospital environment.

The question of whether or not routine HIV testing should be performed is not new. HIV testing is the critical first step to meeting the WHO global strategy goal of having $90 \%$ of HIV-infected individuals tested, $90 \%$ of those on ART, and $90 \%$ of those virally suppressed (90-90-90). ${ }^{[16]}$ Although HIV testing is mandated in all hospitals in SA, patients can refuse testing.

A study performed in Eastern Cape Province, SA, in 2016 $6^{[17]}$ showed that counsellor-initiated, non-targeted universal screening

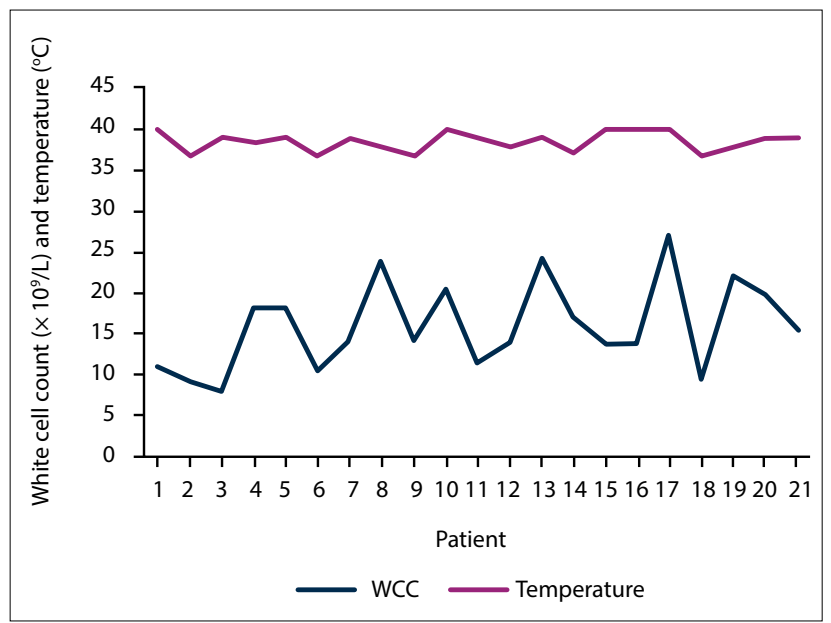

Fig. 1. WCC and temperature of patients $(\mathrm{N}=21)$ who developed nosocomial infections. One patient was referred from another hospital, and primary admission vitals (heart rate and temperature) were unavailable for this patient and were excluded. $($ WCC $=$ white cell count. $)$

Table 1. Sepsis parameters of patients who developed nosocomial sepsis

\begin{tabular}{llll}
\hline & White cell count $(\times \mathbf{1 0} / \mathbf{L})$ & Temperature $\left({ }^{\circ} \mathbf{C}\right)$ & Heart rate $(\mathbf{b p m})$ \\
\hline Mean value (range) & $16.3(7.9-24.3)$ & $38.5(36.7-40.1)$ & $105(54-142)$
\end{tabular}


with rapid point-of-care testing revealed a high HIV prevalence, with a significant burden of undiagnosed HIV infection in the province. Unfortunately, this approach fell short of meeting the testing needs in this setting, with only $26 \%$ of patients being approached by healthcare staff owing to time and resource constraints. ${ }^{[17]}$ It stands to reason that patients with undiagnosed and untreated HIV with high viral loads are predisposed to infection and poor outcomes. With only 2 of the 22 patients in the present study known to be HIV-positive, the numbers were too small to draw any meaningful conclusion regarding association. Continued efforts are needed to educate and encourage routine HIV testing during medical visits.

Preventive strategies performed in our unit include care bundles aimed at reducing the spread of infective organisms. Handwashing between patients and the use of protective gloves and aprons are encouraged, changing of all intravenous access is mandatory upon admission to the ward, and ERAS programmes are implemented. These strategies may explain the low infection rates in the unit.

Initial intervention for sepsis following trauma is challenging, but may improve outcome and decrease mortality. Risk prediction and performance using scoring systems such as the National Nosocomial Infection Surveillance (NNIS) and Efficacy of Nosocomial Infection Control (SENIC) indexes have been proposed to predict SSIs in patients undergoing elective surgery. ${ }^{[14]}$ However, these indexes do not perform well in abdominal trauma patients and an alternative model would need to be validated. ${ }^{[14]}$ Although research on infection in trauma has been investigated, uniform guidelines for antibiotic use after trauma are lacking, particularly following neurosurgical and maxillofacial trauma.

The African Surgical Unit-based Safety Programme was performed between 2013 and 2015 in five African hospitals. ${ }^{[18]}$ This study was the first report based on multicentre SSI surveillance and a multimodal intervention aimed at improving multiple SSI prevention measures in hospitals in sub-Saharan African countries. The interventions included preoperative patient bathing, avoiding hair removal or doing it with clippers, appropriate surgical hand preparation, appropriate patient skin preparation, optimal antibiotic prophylaxis, and improving operating room discipline. The risk of SSI during follow-up was significantly lower than pre-intervention, and a substantial improvement in compliance with prevention measures was consistently observed. ${ }^{[18]}$ This study affirms strategies currently in place at our institution aimed at preventing SSI. Research is underway assessing the effects of ERATS in reducing HAI and improving patient outcomes based on ERAS protocols established for elective colorectal surgery.

\section{Study limitations}

There were several limitations to the present study. No multivariate analysis was performed owing to the descriptive nature of the data collection. A case-control or cohort study is needed in order to perform meaningful statistical analysis and identify whether there were risk factors for the development of nosocomial infections. The number of patients who developed these infections was lower than expected, so our figures may not be a true representation of infection rates. The short study period is another limitation, but February to April are traditionally busy months, during which trauma volume increases after a quieter period in January.

\section{Conclusions}

A combination of measures is needed to prevent trauma-related infection. By determining the incidence of nosocomial infection in our trauma patients, uniform policies to reduce infection rates could be drafted. The low incidence of infection may be explained by preventive care bundles already established.

\section{Declaration. None.}

Acknowledgements. We thank the University of Cape Town and Department of Trauma wards C5 and C12 for allowing us to conduct the research in their institution.

Author contributions. AJD: main author; PHN: manuscript editor; SG: data capture; JCK: author and editor.

Funding. None.

Conflicts of interest. None.

1. Rajkumari N, Sharma K, Mathu P, et al. A study on surgical site infections after trauma surgeries in a tertiary care hospital in north India. Indian J Med Res 2014;140(5):691-694.

2. Nel DC. Surgical site infection. Afr Fam Pract 2014;56(2 Suppl 1):535-539.

3. Morgan A. Risk factors for infection in the trauma patient. J Natl Med Assoc 1992;84(12):1019-1023.

4. Morrison JJ, Yapp L, Beattie A, et al. The epidemiology of Scottish trauma: A comparison of prehospital and in-hospital deaths, 2000 - 2011. Surgeon 2016;14(1):1-6. https://doi.org/10.1016/j. surge.2015.02.001

5. Binkowska A, Michalak G, Slotwinksi R. Current views on the mechanisms of immune responses to trauma and infection. Cent Eur J Immunol 2015;40(2):206-216. https://doi.org/10.5114 ceji. 2015.52835

6. Neumayer L, Hosokawa P, Itani K, et al. Multivariate predictors of surgical site infections after general and vascular surgery: Results from the Patient Safety in Surgery study. J Am Coll Surg 2007;204(6):1178-1187. https://doi.org/10.1016/j.jamcollsurg.2007.03.022

7. Anderson D, Podgorny K, Berríos-Torres S, et al. Strategies to prevent surgical site infections in acute care hospitals: 2014. Infect Control Hosp Epidemiol 2014;35(6):605-627. https://doi org/10.1086/676022

8. Centers for Disease Control and Prevention. Surgical site infection. Guidelines and procedures for monitoring SSI. www.cdc.gov/hai/ssi/ssi.html (accessed 31 December 2018).

9. Horan TC, Gaynes RP, Martone WJ, Jarvis WR, Emori TG. CDC definitions of nosocomial surgical site infections, 1992: A modification of CDC definitions of surgical wound infections. Infect Control Hosp Epidemiol 1992;13(10):606-608.

10. World Health Organization. World report on violence and health. https://www.who.int/violence World Health Organization. World report on violence and health. https://www.who.int
injury_prevention/violence/world_report/en/abstract_en.pdf (accessed 31 December 2018).

11. Ma X-Y, Tian L-X, Liang H-P. Early prevention of trauma-related infection/sepsis. Mil Med Res Ma X-Y, Tian L-X, Liang H-P. Early prevention of
2016;3:33. https://doi.org/10.1186/s40779-016-0104-3

12. Wafaisade A, Lefering R, Bouillon B, et al. Epidemiology and risk factors of sepsis after multiple trauma. Crit Care Med 2011;39(4):621-628. https://doi.org/10.1097/CCM.0b013e318206d3df

13. Morales $\mathrm{CH}$, Escobar RM, Villegas MI, Castaño A. Surgical site infection in abdominal trauma patients: Risk prediction and performance of the NNIS and SENIC indexes. Can J Surg 2011;54(1):17 24. https://doi.org/10.1503/cjs.022109

14. Jin H, Lui Z, Xiao Y, Fan X, Yan J, Liang H. Prediction of sepsis in trauma patients. Burns Trauma 2014;2(3):106-113. https://doi.org/10.4103/2321-3868.135479

15. Tripathi P, Gajbhiye SR, Agrawal GN. Clinical and antimicrobial profile of Acinetobacter spp. An emerging nosocomial superbug. Adv Biomed Res 2014;3:13. https://doi.org/10.4103/22779175.124642

16. Joint United Nations Programme on HIV and AIDS (UNAIDS). 90-90-90: an ambitious treatment target to help end the AIDS epidemic. 2014. https://www.unaids.org/sites/default/files/media_ target to help end the AIDS epidemic. 2014 .

17. Hansoti B, Stead D, Parrish A, et al. HIV testing in a South African emergency department: A missed Hansoti B, Stead D, Parrish A, et al. HIV testing in a South African emergency department:
opportunity. PLoS One 2018;13(3): 0193858 . https://doi.org/10.1371/journal.pone.0193858

18. Allegranzi B, Aiken A, Kubilay N, et al. A multimodal infection control and patient safety intervention to reduce surgical site infections in Africa: A multicentre, before-after, cohort study. Lancet Infect Dis 2018;18(5):507-515. https://doi.org/10.1016/S1473-3099(18)30107-5

Accepted 13 August 2019. 